\section{New fronts against malaria}

\section{By Brian Moy, Staff Writer}

A key challenge in combating malaria is the rapid development of resistance that Plasmodium falciparum parasites mount against older drugs. Now, a pair of articles describes two new ways to attack the infection: inhibiting host calpain activity and inhibiting the parasite's Sir2 histone deacetylases. ${ }^{1,2}$ However, precisely targeting only the calpain of interest could be difficult, whereas compounds against Sir2 would need to be species specific.

In a paper published in Science, researchers at the University of Pennsylvania and colleagues reported that inhibition of calpain 1 (CAPN1) in cultured human erythrocytes blocked $P$. falciparum parasites from escaping infected cells, thus preventing the parasites from proliferating. ${ }^{1}$

Calpains are normally inactive cysteine proteases that become proteolytically active and associate with membranes in the presence of calcium. ${ }^{3}$

To further explore the significance of calpains during parasite infection, the researchers showed that small interfering RNA-mediated suppression or genetic deletion of calpain activity also blocked escape of Toxoplasma gondii parasites from infected mammalian fibroblasts.

"In general, calpains are an unusual target because they are a host target rather than a parasite target. By targeting calpains, the parasite has no way to genetically control the host protein and hopefully would be unable to develop resistance against such a strategy," said Doron Greenbaum, the principal investigator of the study and an assistant professor in the Department of Pharmacology at the University of Pennsylvania School of Medicine.

Next steps for Greenbaum and colleagues include conducting studies in mice to verify that parasitic escape is indeed mediated by host calpains and developing specific calpain inhibitors.

Timothy Wells, CSO at Medicines for Malaria Venture (MMV), noted that developing such compounds could be a challenge. In the past, he said, "it has been quite difficult to only target the cysteine or serine protease of interest without inhibiting all of the others."

He added that "it is unlikely that specific calpain inhibitors will be identified just by screening large collections of compounds." Instead, Wells thinks "the best approach would be to take known inhibitors of calpains from projects in discovery and preclinical stages and determine the effectiveness of those compounds in animal models of malaria."

Greenbaum said the findings of the Science paper are neither patented nor licensed.

\section{Antigenic approach}

A group at The Walter and Eliza Hall Institute of Medical Research took a different tack and opted to attack a parasitic target. In an article published in PLoS Biology, the group reported that inhibiting P. falciparum sirtuin 2 (PF13_0152; Sir2) prevented the parasite from undergoing antigenic variation, a process that helps the bug escape detection by the immune system. ${ }^{2}$

P. falciparum parasites lacking either the Sir2A or Sir2B histone deacetylases showed less switching in expression between the parasite's 60 variants of $P$. falciparum erythrocyte membrane protein 1 (VAR; PfEMP1) than wild-type $P$. falciparum.

Switching amongst PfEMP1 variants is a key mechanism of immune evasion by $P$. falciparum, and PfEMP1 is responsible for cytoadherence of infected cells. Cytoadherence leads to clumping of erythrocytes and blockage of capillaries in the brain, organs and peripheral microvasculature and is a major contributor to morbidity and mortality associated with malaria infection.

According to Alan Cowman, head of the institute's Division of Infection and Immunity and corresponding author on the PLoS Biology paper, infected malaria patients contain on the order of 10 trillion parasites. "This is a huge reservoir for mutation and selection of resistance," he said.

Thus, Cowman said, the group's findings are "a step forward in understanding how Plasmodium falciparum parasites regulate their ability to antigenically vary the surface of the infected erythrocyte. The study provides potential new targets that would interfere with the parasites' ability to undergo antigenic variation and escape the immune system."

Next steps for the researchers include high throughput screening to identify Sir2-specific inhibitors.

MMV's Wells noted that the PLoS Biology study focused on a protein involved in the cytoadherence of erythrocytes in severe malaria. As a result, he said, "there are implications for controlling the acute stages of the disease, which is where fatalities occur."

Peter DiStefano, CSO at Elixir Pharmaceuticals Inc., said that "while sirtuin 2 inhibitors may have potential as stand-alone malaria therapeutics, combination therapy using antimalarial medications and sirtuin 2 inhibitors should also be investigated." He said it will also be important to determine the stage of the parasite's lifecycle at which Sir2 inhibitors would be most effective.

Elixir has a series of human sirtuin 2 (SIRT2) inhibitors in preclinical testing for various neurodegenerative diseases.

Cowman said existing SIRT2 inhibitors "would need to be able to differentially inhibit $P$. falciparum Sir2 proteins and not human sirtuins" to effectively treat malaria.

"In theory we have the technology to develop inhibitors that would target the parasite, but malaria is not one of our focal points, either from an expertise standpoint or a commercial standpoint," DiStefano told SciBX.

Cowman said the group's findings are neither patented nor licensed.

Moy, B. SciBX 2(16); doi:10.1038/scibx.2009.647

Published online April 23, 2009

REFERENCES

1. Chandramohanadas, R. et al. Science; published online April 2, 2009; doi:10.1126/science. 1171085 


\section{TARGETS \& MECHANISMS}

Contact: Doron C. Greenbaum, University of Pennsylvania, Philadelphia, $\mathrm{Pa}$.

e-mail: dorong@upenn.edu

2. Tonkin, C. et al. PLoS Biol.; published online April 14, 2009; doi:10.1371/journal.pbio.1000084

Contact: Alan F. Cowman, The Walter and Eliza Hall Institute of Medical Research, Melbourne, Australia

e-mail: cowman@wehi.edu.au
3. Croall, D. \& Ersfeld, K. Genome Biol. 8, 218 (2007)

COMPANIES AND INSTITUTIONS MENTIONED

Elixir Pharmaceuticals Inc., Cambridge, Mass.

Medicines for Malaria Venture, Geneva, Switzerland

University of Pennsylvania, Philadelphia, Pa.

The Walter and Eliza Hall Institute of Medical Research, Melbourne,

Australia 